\title{
Stepped Leader Progression and Speed Evolution in a Thunderstorm: Theoretical Model ${ }^{\dagger}$
}

\author{
Aníbal Seminario-García ${ }^{1}$, Cristina González-Morán ${ }^{2}\left(\mathbb{1}\right.$ and and Pablo Arboleya ${ }^{2, *}$ (1) \\ 1 Department of Material Science, University of Oviedo, 33204 Gijón, Spain \\ 2 LEMUR Group, Department of Electrical Engineering, University of Oviedo, 33204 Gijón, Spain \\ * Correspondence: arboleyapablo@uniovi.es; Tel.: +34-985182283 \\ + This paper is an extended version of our paper published in 2018 IEEE International Conference on \\ Environment and Electrical Engineering and Industrial and Commercial Power Systems Europe \\ (EEEIC/ICPS Europe), Palermo, Italy, 12-15 June 2018.
}

Received: 22 April 2019 ; Accepted: 19 June 2019; Published: 28 June 2019

\begin{abstract}
This paper presents a theoretical model to describe the progression of leading (falling) lightnings in storms (stepped leaders). Stepped leaders move down from the thundercloud base to the encounter point with an upward streamer. First, the existing models, related to the advance of leading lightnings, are analyzed. Then, a novel theory is presented. The proposed model describes both the leader progression and speed. It aims at explaining the leader progression as a succession of several steps, or branches, that form the well-known tree-like shape. The speed of advance per step is described as a function of various parameters: the charge concentration surface diameter and the step length, among others. The derived formulas include two new parameter named $(\chi)$ and G. $\chi$ is the ratio between the guide beam length $(L)$ and the diameter of the circle, inside the cloud, where the charges are concentrated $(D)$. G relates density of charges, as explained herein.
\end{abstract}

Keywords: electric field; leader progression model; stepped leader; thunderstorm

\section{Introduction}

Electric discharge in long air gaps is a complex phenomenon that has been studied for many different applications [1], one of these applications being the development of mathematical theories able to model the stepper leader progression and its speed evolution in a thunderstorm. Thundercloud consists of several cells, very close to each other but almost independent. A cell is defined as an air region (or volume) that is limited in horizontal and vertical directions. In this limited region, several processes of updrafts and descendants warm and humid air flows occur at the same time ([2] Chapter 9). Due to these movements, and due the Earth's electric field (about $100 \frac{\mathrm{V}}{\mathrm{m}}$ ), the falling water drops have an induced dipole moment. This dipole is positive at the bottom and negative at the top ([2] Chapter 9). On the other hand, there are many large and slow ions in the air, generally produced by friction and collisions between particles of water and ice. If a positive ion approaches the base of the polarized drop, it will be repelled. The ascendant air ends up raising the positive ions to the top of the cloud. In contrast, if a negative ion approaches, it nullifies the positive part of the drop, leaving it negatively charged. As the negative ions are heavier, they end up at the cloud base. In this way, the cloud is negatively charged at the bottom and positive at the top. This mechanism is known as the theory of separation of charges in a thunderstorm. Figure 1 shows a scheme of this mechanism in two steps. The small circles represent the charged ions and $\mathbf{E}$ is the electric field originated in the process. 
stage 1

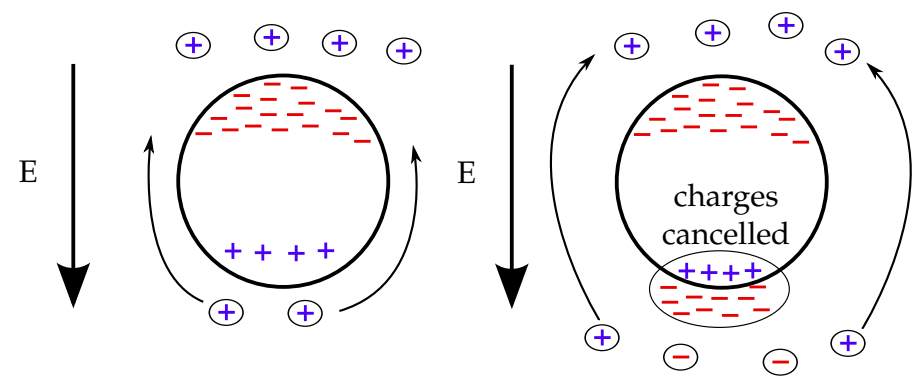

Figure 1. Separation of charges in a thundercloud. Reproduced/Adapted with permission from [3], Anibal Seminario-Garcia, Cristina Gonzalez-Moran, Pablo Arboleya. “Theoretical Model for the Progression of Leader Steppers in a Thundercloud", 2018 IEEE International Conference on Environment and Electrical Engineering and 2018 IEEE Industrial and Commercial Power Systems Europe (EEEIC/ICPS Europe).

The charge separation process creates three types of very intense electric fields (E): inside the cloud, between two different clouds and between the cloud and ground. When the electric field exceeds the local dielectric strength, an electrical discharge occurs in the form of lightning. This channel of ionized air is called leader. Moreover, the leader often splits in multiple branches, reminiscent of a tree form. Then, the channel is called stepped leader. In the case of a cloud-to-Earth discharge, the stepped leader starts at the base of the cloud and progresses as a guide carrying negative charge towards the Earth. When this stepped leader is close to the ground, around 10-200 m approximately, the positive charge of Earth may give rise to a positively ionic channel appearing to meet the leader. This new channel from the ground is called the upward streamer. In Figure 2, the red and blue lines represent the stepped leader and the upward streamer, respectively.

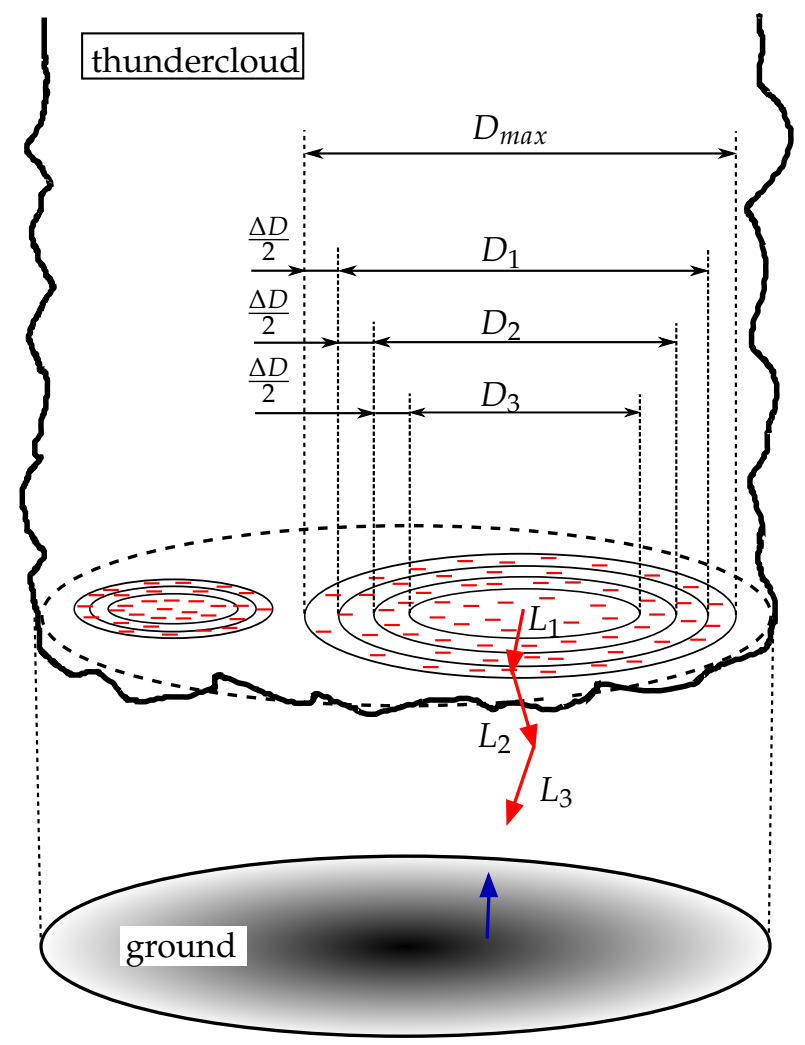

Figure 2. Stepped leader progression in different sections (stages). Reproduced/Adapted with permission from [3]. 
Once the stepped leader and the upstream have met, the lightning channel resistance plummets. Then, the electrons accelerate very quickly and move across the whole leader network at a fraction of the speed of light. This is the so-called return stroke, from ground to cloud. After that, a new stepped leader might appear and the entire process may repeat several times [4-6].

Sometimes, the leader disappears into the atmosphere before it reaches the ground through a process that is explained below.

Related to this work, Dellera, Garbagnati and Bernardi $[7,8]$ developed the leader progression model (LPM). In their studies, they determined the electrical field evolution as the stepped leader advances to meet the upward streamer. They established trajectories that approached quite well to real cases. The trajectories are not straight lines. Their model approaches the cloud to a cylinder, with a diameter of about $10 \mathrm{~km}$, uniformly charged on its base (surface charge is considered). The distance between the cloud and the ground could be near $2 \mathrm{~km}$. According to those considerations, the progression model can be summarized as follows:

- The stepped leader starts from an initial zone, called streamer zone. That is a region where the electrical field is equal or higher than $300 \frac{\mathrm{kV}}{\mathrm{m}}$. The leader advances following always the maximum potential gradient direction (between the streamer zone and the leader tip).

The linear density of charge $\left(\varphi_{l}\right)$, in Coulombs per meter $\left(\frac{\mathrm{C}}{\mathrm{m}}\right)$, can be expressed as a function of the peak intensity $\left(I_{p f}\right)$, in $\mathrm{kA}$ :

$$
\varphi_{l}=38 I_{p f}^{0.68} 10^{-3}
$$

- When the electrical field on the ground (or building, or structure) is equal or higher than the critical value of rupture (about $500 \frac{\mathrm{kV}}{\mathrm{m}}$ ), the upward streamer appears and goes to meet the leader. The upward streamer also follows the direction of the maximum potential gradient.

Rizk [9] described a different stepped leader model, based on a vertical trajectory. According to this author, the trajectory of the stepped leader is not affected by the presence of an upward streamer. The the upward streamer appears when the potential $V(\mathrm{kV})$ of a structure of height $H(\mathrm{~m})$ surpasses the value given by Equation (2).

$$
V=\frac{1556}{1+\frac{3.89}{H}}
$$

In his work, Rizk also claimed that, once the leader and the upward have met, the return stroke is formed, resulting in a peak intensity $I_{p f}$. However, it may occur that they do not really interact. Then, because the leader is faster than the streamer, it impacts on the ground before they meet.

Some authors describe or employ models of stepped leader, and lightnings in general, for protection purposes. Protection against lightning includes transmission lines, electronic equipment, buildings, etc. That is the case of Bank Tavakoli and Vahidi [10] who used the lightning path from cloud to a striking point to study the effect on high voltage overhead lines. They modeled the cloud as ring charges and downward leaders as several steps. In [11], the authors developed a model capable of finding the inception of the upward leaders. The application is related to lightning protection systems for electronic equipment.

The authors of [12] proposed a physical upward leader propagation model. This model is applied to simulate leaders in laboratory experiments. The parameters to describe the model were the leader current, the electric field and the leader speed. In addition, the authors of [13] described a physical model based on experimental investigations. The results show that the step length and the leader speed increase with increasing prospective return stroke current. It also increases as it approaches the ground. The authors of [14] observed several leaders in natural lightning by using a high-speed video camera. The main conclusion is that the speed of leaders changes as they get closer to the ground. In addition, Shah et al. [15] performed a large-scale investigation into leader development in a 10-m rod-lane gap under a long front positive impulse. They recorded the leader propagation with a high-speed charge camera. Long and Becerra [16] studied the lightning attachment of stepped 
leaders to ground objects. Nag and Rakov [17] proposed a unified model to compute the electric field produced by the sequence of the preliminary breakdown, the stepped leader and the return stroke. Wang et al. [18] presented a regional multi-frequency-band lightning detection and location network in China. They applied different location algorithms trying to cover research and operational purposes. In [19], the authors proposed a conceptual view of leader step formation. Zhu et al. [20] used an automated data processing algorithm to study records of electric field for cloud-to-ground flashes reported by the U.S. National Lightning Detection Network.

Regarding the leader speed, Rakov and Uman [21] presented a review of the different theories for leader speed evolution. This review showed the speed is a function of the leader height and can be summarized as follows:

- In 1956, Scholand (South Africa) found speeds near $0.8 \times 10^{5}$ to $8 \times 10^{5} \frac{\mathrm{m}}{\mathrm{s}}$ at a height from the ground of 2 to $3 \mathrm{~km}$.

- In 1961, Isikawa (Japan) measured seeps of $3.1 \times 10^{5} \frac{\mathrm{m}}{\mathrm{s}}$.

- In 1966, Berger and Vogelsanger (Switzerland) got speeds of $0.9 \times 10^{5}$ to $4.4 \times 10^{5} \frac{\mathrm{m}}{\mathrm{s}}$ at a height of approximately $1.3 \mathrm{~km}$.

- In 1982, Overville and Idone (Florida) noticed the leader speed increased near the ground, measuring speeds of $15 \times 10^{5} \frac{\mathrm{m}}{\mathrm{s}}$ at a height of $166 \mathrm{~m}$.

- In 1990, Rakov and Uman found a mean measure of $2 \times 10^{5} \frac{\mathrm{m}}{\mathrm{s}}$.

- In 1999, Chen et al. (Australia) determined $4.5 \times 10^{5}$ to $11 \times 10^{5} \frac{\mathrm{m}}{\mathrm{s}}$ at heights between 367 and $1620 \mathrm{~m}$.

Golde $[22,23]$ computed the leader speed as a function of the lineal density of charge and its length. By deriving that expression, the speed is obtained as a function of its current density. On the other hand, Berger [24] related the upward streamer peak density to the carried charge.

According to the current literature review, more recent works can be added to Rakov and Uman's review [21]:

- In 2006, Becerra and Cooray[12] obtained leader speeds of $0.1 \times 10^{5}$ to $0.5 \times 10^{5} \frac{\mathrm{m}}{\mathrm{s}}$, and observed that leader velocity increases before the final step.

- In 2015, Wang et al. [19] measured speeds of $4.8 \times 10^{5}$ to $5.9 \times 10^{5} \frac{\mathrm{m}}{\mathrm{s}}$ in China.

- In 2016, Wang et al. [18] observed an average leader speed of about $8.7 \times 10^{6} \frac{\mathrm{m}}{\mathrm{s}}$, a decrease in the downward propagation speed.

- In 2018, Shah et al. [15] measured experimental speeds of $0.1 \times 10^{5}$ to $0.6 \times 10^{5} \frac{\mathrm{m}}{\mathrm{s}}$.

The new theoretical model proposed in this paper explains the progression of stepped leaders and the evolution of their speed. This work improves the model presented in [3] and includes a deeper study of the leader progression and a new detailed model of its speed.

In this case, we include a deeper study on leader trajectories and a new study of their speed. Because the leader has been demonstrated to advance into different steps, we have explained the leader progression as several sections of the whole trajectory. At each step, the direction is fixed by the electrical resistance offered by the air mass. The proposed model also describes the leader speed. The speed variation is presented as a function of several parameters, such us diameter of the region of concentration of charges in the thundercloud, among other atmospheric parameters. The main novelty of this proposal is that it is completely theoretic, and it describes and adapts to most of the theories previously described, reinforcing some of them. The speed model is based on a new parameter, named $\chi$. It defines the ratio between the length of advance and the diameter of the surface of concentration of charges. We demonstrate that this new parameter plays an important role in the leader speed evolution.

In the following sections, we explain the mathematical model for stepped leader evolution in different sections and its speed variation as well. Then, a section to compared the work presented in here to recent studies is included. In the last section, the conclusion is presented. 


\section{Mathematical Model of the Leader Progression}

\subsection{Problem Statement}

The mathematical model is based on the following considerations: The whole process starts inside an already formed thundercloud. Then, the negative charge surface, located at the cloud bottom, is approached as several concentric circles. This description is represented in Figure 2. $D_{\max }$ stands for the initial diameter of the outer circle. Assuming that the maximum initial charge is $Q_{\max }$ in Coulombs, the surface density or charge $\left(\varphi_{s}\right)$ can be expressed as:

$$
\varphi_{s}=\frac{Q_{\max }}{\pi \cdot \frac{D_{\max }^{2}}{4}}
$$

Then, the process evolves in this way: In the first stage, there is a partial progression of the stepped leader. As the leader advances, the section of the initial negative charged circle decreases. For example, if the leader advances a length of $L_{1}$, the diameter changes to $D_{1}$. The total decrease in this diameter can be expressed as (see Figure 2):

$$
\Delta D=D_{\max }-D_{1}
$$

The charge delivered by the leader is calculated as the initial charge (the maximum charge at the beginning, in the first step, from Equation (3)) minus the remaining charge at the cloud. First, the maximum initial charge is computed as:

$$
Q_{\max }=\varphi_{s} \cdot \pi \cdot \frac{D_{\max }^{2}}{4}
$$

Then, the remaining charge can be computed using Equation (3), but replacing $D_{\max }$ by $D_{1}$ (the latter is obtained from Equation (4)):

$$
q_{1}=\varphi_{s} \cdot \pi \cdot \frac{\left(D_{\max }-\Delta D\right)^{2}}{4}
$$

Finally, the charge delivered by the stepped leader is obtained as the difference between Equations (5) and (6):

$$
q_{L_{1}}=\frac{\varphi_{s} \pi}{4} \cdot \Delta D \cdot\left(2 D_{\max }-\Delta D\right)
$$

Since the step length and the charge can be related through the longitudinal density of charge:

$$
\varphi_{L}=\frac{q_{L}}{L}
$$

the leader length of advance in the first step is computed as:

$$
L_{1}=\frac{\pi \cdot \varphi_{s}}{4 \cdot \varphi_{L}} \Delta D \cdot\left(2 \cdot D_{\max }-\Delta D\right)
$$

After the first step, the leader advances in successive stages $L_{2}, L_{3}, \cdots, L_{n}$ in which the diameters change to $D_{2}, D_{3}, \cdots, D_{n}$ respectively. For each step, the previously described procedure is applied to calculate the remaining charge using Equation (6). For instance, in a second step, the leader advances $L_{2}$ :

$$
L_{2}=\frac{\pi \cdot \varphi_{s}}{4 \cdot \varphi_{L}} \Delta D \cdot\left(2 \cdot D_{\max }-3 \cdot \Delta D\right)
$$


Assuming that the total number of steps is $n$, the final expression (that can be considered as a general expression for a given step) is of the following form:

$$
L_{n}=\frac{\pi \cdot \varphi_{s}}{4 \cdot \varphi_{L}} \Delta D \cdot\left(2 \cdot D_{\max }-(2 n-1) \cdot \Delta D\right)
$$

This procedure is applied to several steps, until the leader reaches the ground, or an upward streamer. Considering constant linear density of charge [25], the total length covered by the stepped leader $(L)$ may be obtained as the sum of the partial lengths.

$$
\begin{array}{r}
L=\frac{\pi \cdot \varphi_{s}}{4 \cdot \varphi_{L}} \Delta D \cdot\left[2 \cdot D_{\max }-\Delta D+\ldots+2 \cdot D_{\max }-3 \cdot \Delta D+2 \cdot D_{\max }-\ldots\right. \\
\left.\ldots-5 \cdot \Delta D+2 \cdot D_{\max }-(2 n-1) \cdot \Delta D\right]
\end{array}
$$

Rearranging terms in Equation (13), it can be written:

$$
L=\frac{\pi \cdot \varphi_{s}}{4 \cdot \varphi_{L}} \cdot \Delta D \cdot\left[2 n \cdot D_{\max }-(1+3+5+\ldots+n-1) \cdot \Delta D\right]
$$

The sum $(1+3+5+\ldots+n-1)$, in Equation (13), describes an arithmetic progression of odd numbers. Thus, it can be substitute by $n^{2}$. In this way, a more compact equation can be rewritten:

$$
L=\frac{\pi \cdot \varphi_{s}}{4 \cdot \varphi_{L}} n \cdot \Delta D \cdot\left(2 \cdot D_{\max }-n \cdot \Delta D\right)
$$

The minimum diameter, at the end of the process, can be computed as:

$$
D_{\min }=D_{\max }-n \cdot \Delta D
$$

From this expression, we can obtain $n \cdot \Delta D$ and replace its value in Equation (14). Thus, it can be written:

$$
L=\frac{\pi \cdot \varphi_{s}}{4 \cdot \varphi_{L}} \cdot\left(D_{\max }^{2}-D_{\min }^{2}\right)
$$

Equation (16) describes the stepped leader behavior when it meets an upward streamer but also if the leader does not reach the ground. In the former case, the thundercloud remains charged, thus allowing subsequent processes, being the final diameter $D_{\min }>0$. In the latter case, the leader has already carried all charge available, being the final diameter $D_{\min }=0$ before it reaches the ground.

The stepped leader trajectory would be a straight line if the air would not offer electrical resistance. In that case, the leader would move at the speed of light. However, experiments reveal the real trajectories are zigzags. The total length of advance might be much larger than the distance between cloud and ground. Besides, the speed is less than a third of the speed of light [21].

\subsection{Description of the Stepped Leader Trajectory}

Equation (16) shows us how the derived trajectory approaches to real cases. For that propose, let us consider a trajectory split in three different steps. In Figure 3, a diagram representing this assumption is depicted. Under this considerations, the three steps are established by the following equations:

$$
\begin{aligned}
& L_{1}=\frac{\pi \cdot \varphi_{s}}{4 \cdot \varphi_{L}} \cdot\left(D_{\text {max }}^{2}-D_{1}^{2}\right) \\
& L_{2}=\frac{\pi \cdot \varphi_{s}}{4 \cdot \varphi_{L}} \cdot\left(D_{1}^{2}-D_{2}^{2}\right) \\
& L_{3}=\frac{\pi \cdot \varphi_{s}}{4 \cdot \varphi_{L}} \cdot\left(D_{2}^{2}-D_{3}^{2}\right)
\end{aligned}
$$


The direction followed by the leader in the first step could be any of those in the geometrical place depicted in Figure 3. That is sphere of radius $L_{1}$, as shown in the picture. The proper choice is the way of minimum electrical resistance offered by air. Then, for the second step, $L_{2}$ is the length covered by the leader. Again, the geometrical place for all possible trajectories is a sphere of radius $L_{2}$. The final choice is the path with the lowest resistance. Another step of length $L_{3}$ is also depicted. These three steps define the whole trajectory in this case. The sum of the three partial lengths gives the total length (Equation (16)).

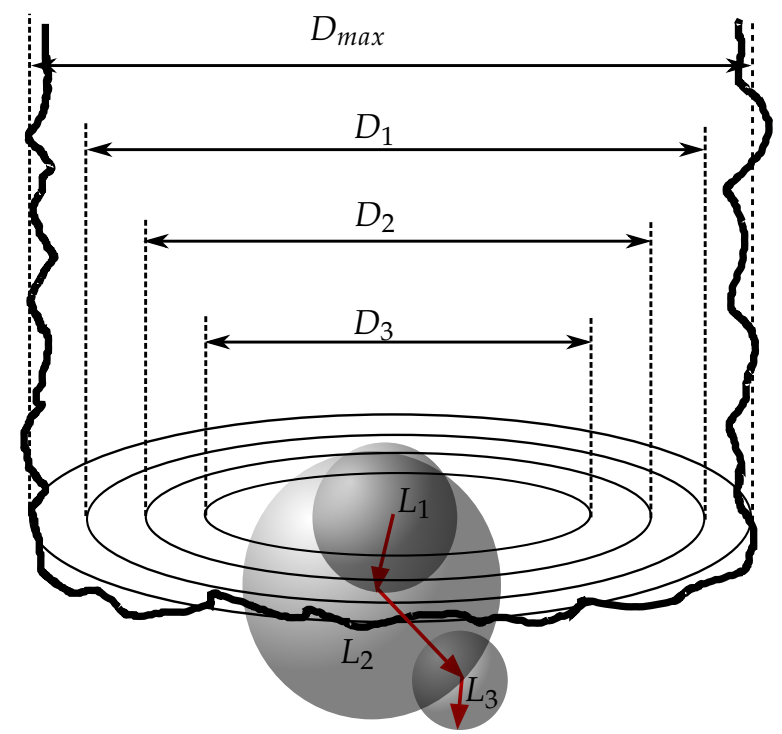

Figure 3. Stepped leader progression sections.

The last step, the third in this example, can give rise to two different possibilities:

(1) The leader meets an upward streamer. Together, they give rise to several subsequent discharges until the thundercloud charge is canceled (it disappears).

(2) The leader carries all the remaining charge in its last step, $L_{3}$. In this case, the third diameter is zero $D_{3}=0$. The length of the last step is computed as:

$$
L_{3}=\frac{\pi \cdot \varphi_{s}}{4 \cdot \varphi_{L}} D_{2}^{2}
$$

Summarizing, the stepped leader trajectory can be described as a sequence of several steps in a zigzag, until the charge from the cloud dissipates or the leader meets an upward streamer.

With this model, it has been demonstrated that the length covered by the stepped leader is directly proportional to the superficial density of charge and inversely proportional to the linear density of charge carried by the leader. The proportionality factor is defined by the squared difference between the initial and the final diameters of the thundercloud base. The final diameter might be zero, in the case the leader does not meet an upward streamer. In other words, there is no remaining charge to be carried, so the leader has dissipated.

\section{Mathematical Model for the Leader Speed}

\subsection{Problem Statement}

The leader speed can be computed through the theoretical electric intensity of the descendant stepped leader. This intensity can be obtained in two different ways that are about to be described. In both cases, the couple cloud-ground is approached by a a large cylindrical capacitor. The charges 
are concentrated on imaginary circular plates, located, respectively, at certain points in the cloud and Earth (see Figure 4).

In the first case, the electrical charge has to be considered in both plates. The positive charge $\left(q^{+}\right)$ is concentrated in plate B, while the negative charge is located in plate $A\left(q^{-}\right)$(see Figure 4). Then, we consider the well known expression with the current intensity $(I$, in $A)$, depending on the capacitance $(C$, in $\mathrm{F})$ and the potential variation with respect to time $\left(\frac{d \phi_{A B}}{d t}\right.$, in $\left.\frac{\mathrm{V}}{\mathrm{s}}\right)$ (Equation (18)). In this particular case, the capacitance represents the capacitor between cloud and ground, the potential is defined between the two plates and the current density is the carried current.

$$
I=\frac{1}{2} \cdot C \cdot \frac{d \phi_{A B}}{d t}
$$

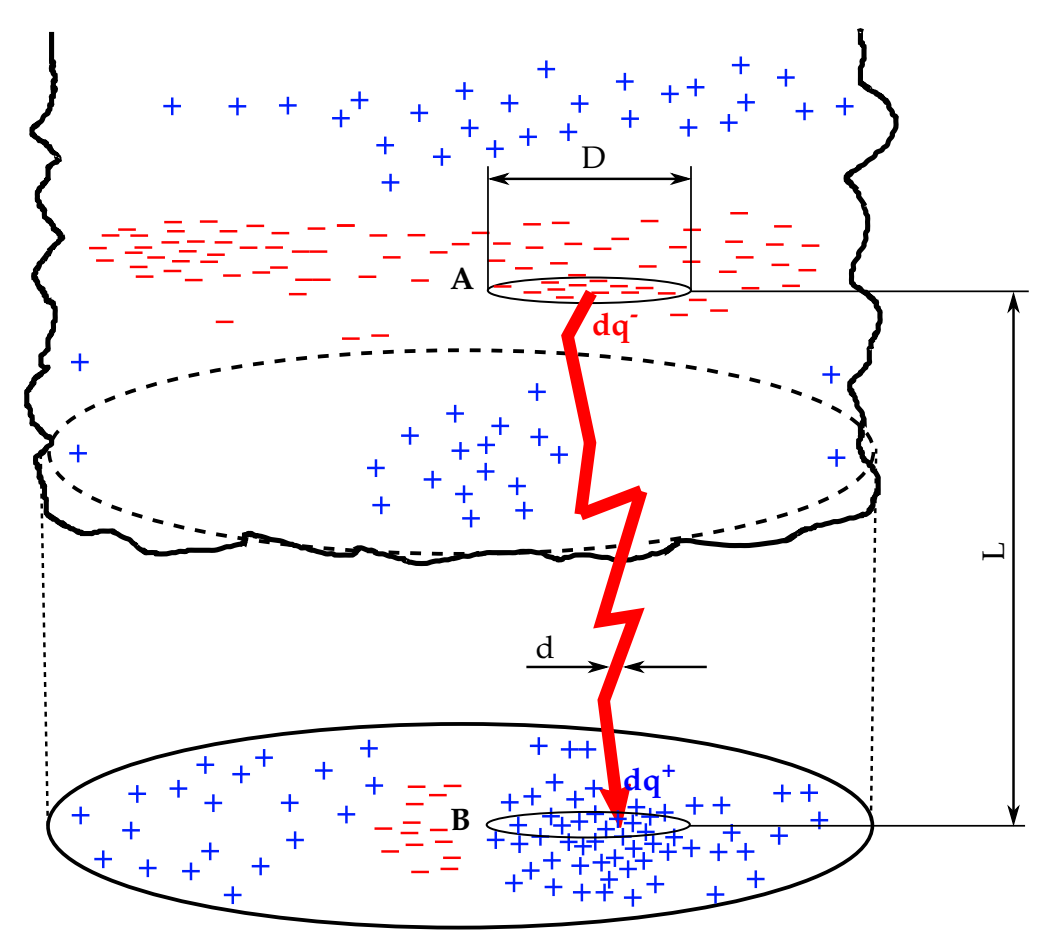

Figure 4. Distribution of charges.

The capacitance $(C)$ is computed through the vacuum permittivity $\varepsilon_{0}=8.85 \cdot 10^{-12} \frac{\mathrm{C}^{2}}{\mathrm{~N} \cdot \mathrm{m}^{2}}$, the diameter $(D$, in $\mathrm{m}$ ) of the imaginary plate inside the cloud and $L$ (in $\mathrm{m}$ ) the length covered by the stepped leader. For simplicity, it is approached as the distance between the cloud and the ground.

$$
C=\varepsilon_{0} \cdot \frac{\pi \cdot D^{2}}{4 \cdot L}
$$

Equation (18) can be transformed by multiplying and dividing the right term by $d l$ and substituting the capacitance by Equation (19). Then, we obtain and expression including the derivative of length with respect to time $\left(\frac{d l}{d t}\right)$ :

$$
I=\frac{1}{2} \cdot C \cdot \frac{d \phi_{A B}}{d l} \cdot \frac{d l}{d t}
$$

Considering that the derivative of the potential with respect to the length is equal to the electrical field $\left(E_{A B}\right.$, in $\left.\frac{\mathrm{V}}{\mathrm{s}}\right)$, and the derivative of the length with respect to time is the leader speed $\left(v\right.$, in $\left.\frac{\mathrm{m}}{\mathrm{s}}\right)$, 
we obtain the equation that relates the discharge intensity of the capacitor that consists of two plates, $\mathrm{A}$ and $\mathrm{B}$, which correspond to cloud and ground, respectively.

$$
I=\varepsilon_{0} \cdot \frac{\pi \cdot D^{2}}{8 \cdot L} \cdot E_{A B} \cdot v
$$

In the second case, the lightning discharge is considered as a resistive electrical circuit. According to Ohm's law, the current intensity is:

$$
I=\frac{\phi_{A B}}{R}
$$

where $R$ is the electrical Ohmic resistance, which depends on the material and its resistivity $(\delta$, in $\Omega \mathrm{m})$, the leader length $(L$, in $\mathrm{m})$ and the leader cross section $\left(s\right.$, in $\left.\mathrm{m}^{2}\right)$. In turn, the cross section is a function of the length $(L)$ and the diameter $(d)$, both represented in Figure $4: s=\frac{\pi \cdot d^{2}}{4}$. Thus, the resistance can be written as:

$$
R=\delta \cdot \frac{4 \cdot L}{\pi \cdot d^{2}}
$$

If we replace Equation (23) into Equation (22), the current intensity is obtained as a function of the stepped leader length and diameter:

$$
I=\frac{\pi \cdot d^{2}}{4 \cdot \delta \cdot L} \cdot \phi_{A B}
$$

In this equation, the relationship between potential and length might be replaced by the electric field: $E_{A B}=\frac{\Phi_{A B}}{L}$. The obtained expression gives us the current as a function of the leader diameter, its total length and the electric field. Considering this expression together with Equation (21), we propose the hypothesis:

$$
\varepsilon_{0} \cdot \frac{\pi \cdot D^{2}}{8 \cdot L} \cdot E_{A B} \cdot v=\frac{\pi \cdot d^{2}}{4 \cdot \delta} \cdot E_{A B}
$$

Finally, the leader speed is obtained as:

$$
v=\frac{2 \cdot d^{2} \cdot L}{\varepsilon_{0} \cdot \delta \cdot D^{2}}
$$

This equation needs two corrections.

- The first one has to do with Lorentz-Fitzgerald contraction that establishes that the length of an element in movement is lower than in rest. Without this contraction, it could happen that the leader reaches speeds higher than the speed of light, as proved below. This situation would be against the relativistic theory. The Lorentz-Fitzgerald contraction states that:

$$
l=l_{0} \cdot \sqrt{1-\frac{v^{2}}{C_{L}^{2}}}
$$

where $l_{0}$ is the length of the element in rest, $l$ is the length in movement, $v$ is the speed of movement and $c_{L}$ is the speed of light.

- On the other hand, the resistivity can be expressed as a function of the environment dielectric strength and the material dielectric constant. To do so, the resistivity is obtained from Equation (24), multiplying and dividing by $L$ as:

$$
\delta=\frac{\frac{\phi_{A B}}{L}}{\frac{I}{L}} \cdot \frac{\pi \cdot d^{2}}{4 \cdot L}
$$


The relationship $\frac{\phi_{A B}}{L}$ is the environment dielectric strength $E_{R D}$, in $\left(\frac{\mathrm{V}}{\mathrm{m}}\right)$, while $\frac{I}{L}$ is the dielectric constant of the material $k_{D}$, in $\frac{\mathrm{A}}{\mathrm{m}}$. The new expression of $\delta$ is:

$$
\delta=\frac{E_{R D}}{K_{D}} \cdot \frac{\pi \cdot d^{2}}{4 \cdot l}
$$

Combining Equations (4), (26) and (27), we obtain:

$$
v=\frac{8 \cdot K_{D} \cdot l_{0}^{2} \cdot\left(1-\frac{v^{2}}{c_{L}^{2}}\right)}{\pi \cdot \varepsilon_{0} \cdot E_{R D} \cdot D^{2}}
$$

For the sake of simplification, from now on, we express the leader length $l_{0}$ without the subindex.

For the next step in this reasoning, we define the parameter $\chi$ as the ratio between the leader length $(L)$ and the diameter of the cloud $(D)$. Thus, $\chi$ results dimensionless:

$$
\chi=\frac{L}{D}
$$

$\lambda$, in $\Omega$, is the resistance that the atmosphere offers against the leader advance.

$$
\lambda=\frac{E_{R D}}{K_{D}}
$$

With these two assumptions, we can rewrite Equation (30) as:

$$
v^{2}+\frac{\pi \cdot \varepsilon_{0} \cdot c_{L}^{2} \cdot \lambda}{8 \cdot \chi^{2}} \cdot v-c_{L}^{2}=0
$$

To solve $v$ from this second-order equation, only the real root is taken. In this way, the leader speed is obtained:

$$
v=\frac{\pi \cdot \varepsilon_{0} \cdot c_{L}^{2}}{16} \frac{\lambda}{\chi^{2}}\left[-1+\sqrt{1+\left(\frac{16}{\pi \cdot \varepsilon_{0} \cdot c_{L}}\right)^{2}\left(\frac{\chi^{2}}{\lambda}\right)^{2}}\right]
$$

To simplify Equation (34), two constants $a$ and $b$ are computed as a function of $\pi, \varepsilon_{0}$ and $c_{L}$. The simplified equation is:

$$
v=a \cdot \frac{\lambda}{\chi^{2}}\left[-1+\sqrt{1+b\left(\frac{\chi^{2}}{\lambda}\right)^{2}}\right]
$$

where $a=15.64 \cdot 10^{4}$, in $\frac{\mathrm{m}}{\mathrm{s} \Omega}$ and $b=368 \cdot 10^{4}$, in $\Omega^{2}$.

In Figure 5, the leader speed is represented as a function of the parameter $\chi$, for different values of $\lambda$. For each $\lambda$, two different lines are plotted: solid lines include the Lorenz contraction while dotted lines do not. This proves that, without considering the Lorenz contraction, leader speeds higher than the speed of light would be obtained.

A deeper analysis of Equation (35) and Figure 5 shows that, for a given $\chi$, when $\lambda$ tends to zero, the speed will reach values near the speed of light $\left(v \rightarrow a \sqrt{b}=3 \cdot 10^{8} \mathrm{~m} / \mathrm{s}\right)$. On the other hand, when $\lambda \rightarrow \infty$, the speed approaches to zero $v \rightarrow 0$. 


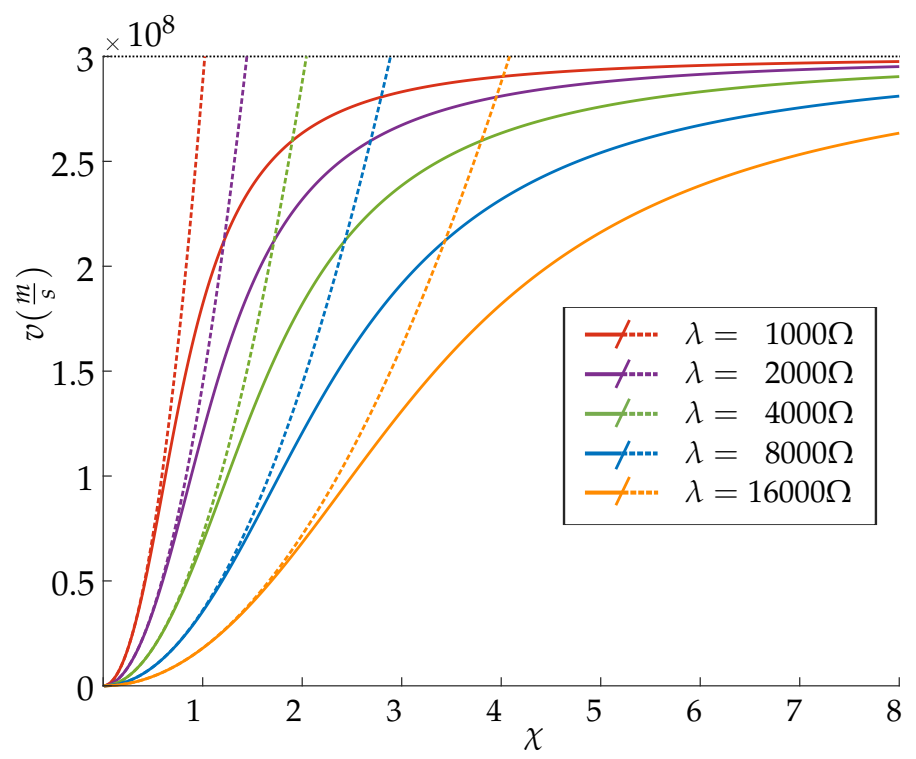

Figure 5. Stepped leader speed as a function of $\chi=\frac{L}{D}$ Solid lines correspond to Lorenz contraction.

\subsection{Analysis of the Leader Speed}

The obtained results show the influence of the environmental resistance and $\chi$ on the leader speed. The parameters $\lambda$ and $\chi$ may vary during the advance of the stepped leader. It is observed that, for the same value of $\chi$, as the resistance increases, the leader speed decreases. On the other hand, if the resistance $\lambda$ is kept constant, as the ratio $\chi$ increases, so does the leader speed. For very high values of $\chi$, this speed might approach the speed of light.

If we consider now the $\chi$ parameter at a give time instant, it represents the variation of the leader length $(l)$ with the plate diameter $(D)$ :

$$
\chi=\frac{d l}{d D}
$$

Solving this differential equation to get the total length:

$$
L=\chi \cdot\left(D_{\max }-D_{\min }\right)
$$

If we substitute this length into Equation (16), $\chi$ is expressed as a function of the leader density of charge, the plate density of charge, and the initial and final diameters of the plates:

$$
\chi=\frac{\pi \cdot \varphi_{s}}{4 \cdot \varphi_{l}} \cdot\left(D_{\max }+D_{\min }\right)
$$

Furthermore, with the mean diameter, defined as the mean value considering the maximum and the minimum diameters $\left(D_{\text {mean }}=\frac{1}{2}\left(D_{\max }+D_{\text {min }}\right)\right)$, we obtain:

$$
\chi=\frac{\pi \cdot \varphi_{s}}{2 \cdot \varphi_{l}} \cdot D_{\text {mean }}
$$

From now on, the sub-index "mean" is removed from the equation, to simplify the expressions. Analyzing this equation together with Figure 3, it is deduced that, for each different step of the leader progression, between two different diameters $D_{i}$ and $D_{j}$, the parameter $\chi$ varies depending on the mean value $D=\frac{1}{2}\left(D_{i}+D_{j}\right)$. This expression also allows removing the $\chi$ parameter from Equation (34). The new equation gives a different perspective of the leader speed. 


$$
v=\frac{\varepsilon_{0} \cdot c_{L}^{2} \cdot \varphi_{l}^{2}}{4 \cdot \pi \cdot \varphi_{s}^{2}} \frac{\lambda}{D^{2}}\left[-1+\sqrt{1+\frac{c_{L}^{2}}{\frac{\varepsilon_{0}^{2} \cdot c_{L}^{4} \cdot \varphi_{l}^{4}}{4^{2} \cdot \pi^{2} \cdot \varphi_{s}^{4}}} \frac{D^{4}}{\lambda^{2}}}\right]
$$

A parameter $G$, in $\left(\frac{\mathrm{m}}{\mathrm{s} \Omega / \mathrm{m}^{2}}\right)$, might be defined as:

$$
G=6.34 \cdot 10^{4} \cdot\left(\frac{\varphi_{l}}{\varphi_{s}}\right)^{2}
$$

This parameter represents, in fact, the amount of charge carried by the leader by the ohmic resistance that the atmosphere offers $\left(\frac{\mathrm{m}^{3}}{\mathrm{~s} \Omega}\right)$. It might be also defined as the relationship between the leader speed and the atmosphere ohmic resistance per squared meter. Using this parameter $G$, Equation (40) can be simplified:

$$
v=G \cdot \frac{\lambda}{D^{2}} \cdot\left[-1+\sqrt{1+\frac{c_{L}^{2}}{G^{2}} \frac{D^{4}}{\lambda^{2}}}\right]
$$

In a more compact form:

$$
v=\frac{c_{L}^{2} \cdot D^{2}}{G \cdot \lambda+\sqrt{G^{2} \cdot \lambda^{2}+c_{L}^{2} \cdot D^{4}}}
$$

In Figure 6, the variation of the leader speed as a function of the cloud plate diameter is represented for different values of $\frac{\varphi_{l}}{\varphi_{s}}$. It has been demonstrated that, as the diameter $(D)$ increases, the step leader speed increases as well. On the other hand, if the resistance of the environment $(\lambda)$ becomes greater, the curve would lie down and acquire lower speeds for the same diameter.

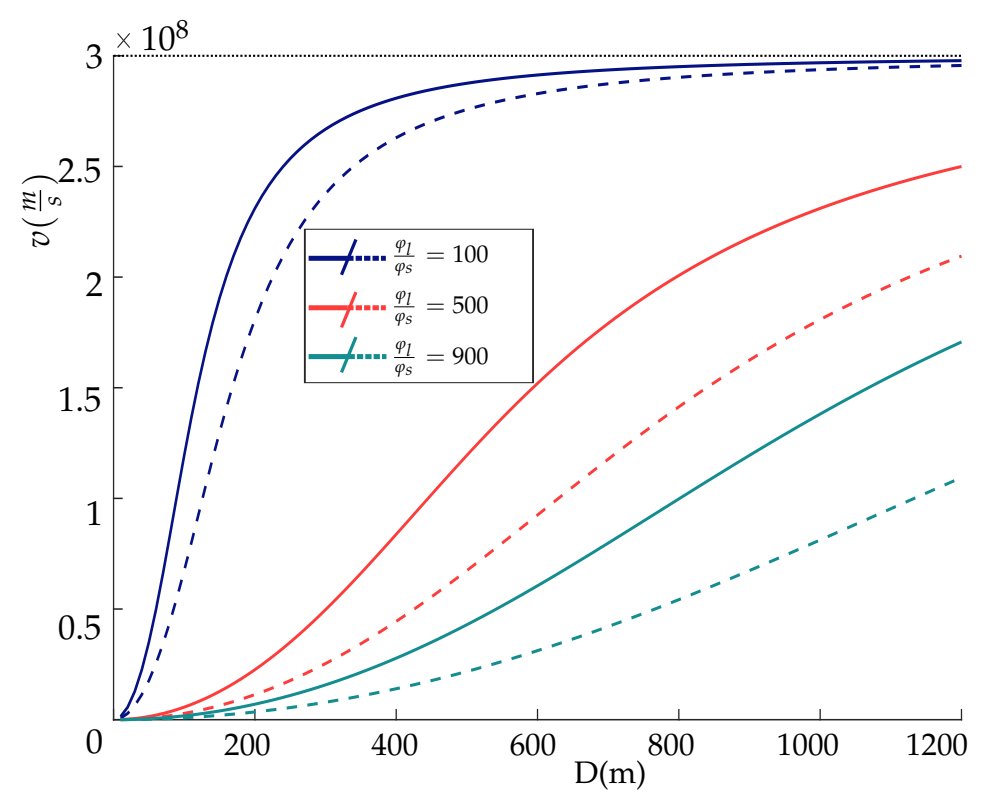

Figure 6. Steeped Leader Speed as function of the diameter for different $\frac{\varphi_{l}}{\varphi_{s}}$. Solid lines are for $\lambda=5000 \Omega$ while dotted lines are for $\lambda=10,000 \Omega$.

The $G$ parameter is an indicator of the leader discharge capacity:

- When $G$ tends to zero, the leader speed approaches the speed of light. This fact is due to a surface charge density, inside the cloud, much higher than the linear density of leader. This situation implies a fast discharge, forcing the leader to acquire a speed close to light, thus reducing the cloud load very quickly. 
- When $G$ is very high, the lightning speed tends to be small due to the large amount of load carried by the leader, forcing it to go slowly. In this case, the linear charge density is much higher than the surface charge density.

It should be noted that the speed range $0.5 \times 10^{5}-20 \times 10^{5} \frac{\mathrm{m}}{\mathrm{s}}$, found by several authors (see Introduction section), is considered for environmental resistances higher than $10,000 \Omega$ and with a load density ratio $\left(\frac{\varphi_{l}}{\varphi_{s}}\right)$ well above $1000 \mathrm{~m}$.

To describe how the leader speed evolves, we present an example in Figure 7. We start from point A with an initial diameter $D_{1}$. The stepped leader starts its progression with a speed of $v_{1}$ imposed by the atmospheric conditions $\lambda_{1}$ and $G_{1}$. As the diameter decreases, the speed also decreases because it follows the trajectory of the $\mathrm{AB}$ curve. However, it may happen, in $\mathrm{B}$, that the values of $\lambda_{1}$ and $G_{1}$ change to $\lambda_{2}$ and $G_{2}$, thus we move to point $C$. This new situation causes the speed to increase. Then, the diameter decreases through the line corresponding to $\lambda_{2}$ and $G_{2}$. If these values of $\lambda_{2}$ and $G_{2}$ are kept constant from this point onwards, the diameter would decrease according to the $C D$ path. Being at point $\mathrm{D}$, the environmental conditions may vary again, reaching, for example, point $\mathrm{E}$ and so on, until the diameter in the cloud disappears.

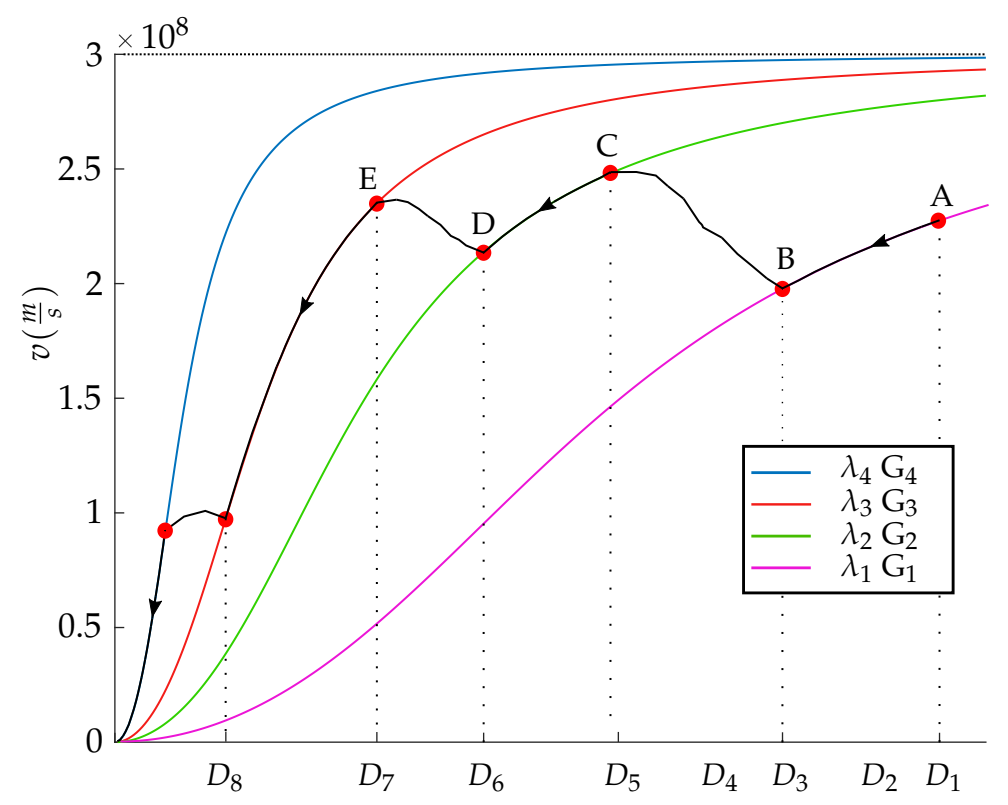

Figure 7. Stepped Leader speed evolution.

\section{Comparison to Recent Studies}

In Table 1, a comparison to the most relevant among the recent studies is shown. The information is organized considering two models, the progression itself on the one side, and the leader speed on the other. For each work, a short description of the contributions to both models and the involved parameters is shown. In our work, the theoretical contribution is reflected in the definition of parameters $\chi$ and $G$, which were not previously employed. It has been demonstrated that both parameters play important roles in describing stepped leader behavior. 
Table 1. Comparison to previous studies.

\begin{tabular}{|c|c|c|c|c|}
\hline References & Progression Model & $\begin{array}{l}\text { Progression } \\
\text { Parameters }\end{array}$ & Speed Model & Speed Parameters \\
\hline$[12,16]$ & $\begin{array}{l}\text { Attachment of dart and } \\
\text { dart-stepped leaders to } \\
\text { ground objects }\end{array}$ & $\begin{array}{l}\text { Derivative of charge } \\
\text { with linear density } \\
\text { of charge }\end{array}$ & $\begin{array}{l}\text { Complex function with } \\
\text { several parameters }\end{array}$ & $\begin{array}{l}\text { Input energy, } \\
\text { time constant, } \\
\text { temperature }\end{array}$ \\
\hline [15] & Experimental & Voltage, time & Experimental & Voltage, time \\
\hline [10] & $\begin{array}{l}\text { Cloud as ring charges. } \\
\text { Leader in steps. }\end{array}$ & $\begin{array}{l}\text { Peak current, cloud } \\
\text { and leader heights, } \\
\text { charge density }\end{array}$ & - & - \\
\hline [13] & Leader in steps & Length, current & $\begin{array}{l}\text { Function of some } \\
\text { parameters }\end{array}$ & $\begin{array}{l}\text { Peak current, height } \\
\text { to the tip }\end{array}$ \\
\hline This work & $\begin{array}{l}\text { The cloud transfers } \\
\text { charge to the leader. } \\
\text { As the leader advances } \\
\text { the cloud diameter } \\
\text { decreases. }\end{array}$ & $\begin{array}{l}\text { Charge densities. } \\
\text { Maximum and } \\
\text { minimum diameters }\end{array}$ & $\begin{array}{l}\text { The couple cloud-ground } \\
\text { is a capacitor that } \\
\text { discharges through a } \\
\text { resistive circuit }\end{array}$ & $\begin{array}{l}\text { Cloud diameter, air } \\
\text { resistance, } \chi, G\end{array}$ \\
\hline
\end{tabular}

\section{Conclusions}

In this paper, we present a mathematical model to explain the behavior of stepped leaders advance in a thundercloud and their speeds. The trajectories are split into different sections, which are reminiscent of the typical tree-form of leaders. The cloud is approached as a cylinder. The base diameter is one the most important parameters to be considered. With each step of advance, this diameter changes. The model establishes the length of each step as a function of several parameters: the diameters at the beginning and end of the step, the surface charge density, the initial charge in the cloud base and the linear charge density carried by the stepped leader. The linear and surface charge densities are assumed to remain constant during the whole process. The obtained equations describe the behavior for the different stepped leader trajectories. The model is appropriated if the leader meets an upward streamer but also if it impacts on the ground.

If the air does not offer electrical resistance, the path would be vertical (straight trajectory, to cover the minimum distance), but real cases have demonstrated the paths are not straight: The leader follows a tree-like form trajectory, and the total might be higher than the distance from the cloud to the ground itself. This fact justifies propagation speeds no higher than a third of the speed of light [21]. The propose mathematical model also matches this tree-like trajectories followed by stepped leader lightnings in a thundercloud.

When the leader starts its progression, it starts at a maximum speed that will decrease as the diameter inside the cloud decreases. This situation may not occur all the time, thus the initial speed approaches light or simply acquires a very small speed that can be observed by the human eye. The parameters that determine this behavior are:

- $\quad \chi$ parameter at a give time instant. It represents the variation of the leader length $(l)$ with the plate diameter $(D)$. As $\chi$ increases so does the leader speed.

- Ohmic resistance of the environment $\lambda$ : The lower is the resistance, the faster is the speed. The maximum atmosphere resistance is determined by dividing the air dielectric strength by the dielectric constant $(30,000 \Omega)$.

- Parameter G. G represents the squared ratio, between the leader linear density of charge and the cloud surface density of charge. When $G$ approaches zero, the linear density is very small compared to the surface density. In this case, it the leader speed approaches the speed of light, alleviating the cloud concentration of charges. Conversely, if $G$ is very large, the leader tends to go slowly due to the large amount of charge transported. 
Author Contributions: A.S.-G. developed the idea and the electrical models; C.G.-M. structured the mathematical formulation; and P.A. reviewed the coherence of the models.

Funding: This work has been supported by Principality of Asturias Government, Spain, under grant FC-GRUPINIDI/2018/000241.

Conflicts of Interest: The authors declare no conflict of interest.

\section{References}

1. Beroual, A.; Fofana, I. Discharge in Long Air Gaps; IOP Publishing: Bristol, UK, 2016; pp. 2053-2563. [CrossRef]

2. Feynman, R.; Leighton, R.B.; Sands, M. The Feynman Lectures on Physics: Mainly Electromagnetism and Matter; Pearson Education: Upper Saddle River, NJ, USA, 1998; Volume 2.

3. Seminario-Garcia, A.; Gonzalez-Moran, C.; Arboleya, P. Theoretical Model for the Progression of Leader Steppers in a Thundercloud. In Proceedings of the 2018 IEEE International Conference on Environment and Electrical Engineering and 2018 IEEE Industrial and Commercial Power Systems Europe (EEEIC/I CPS Europe), Palermo, Italy, 12-15 June 2018; pp. 1-4. [CrossRef]

4. Williams, E.R. The Electrification of Thunderstorms. Sci. Am. 1988, 259, 88-99. [CrossRef]

5. Torres-Sanchez, H. El rayo: Mitos, Leyendas, Ciencia y Tecnologia; Universidad Nacional de Colombia: Bogotá, Colombia, 2002. (In Spanish)

6. Dwyer, J.R. El Rayo. In Investigacion y Ciencia (Spanish Edition of Scientific American); Prensa Cientifica S.A.: Barcelona, Spain, 2005; Volume 346. (In Spanish)

7. Bernardi, M.; Dellera, L.; Garbagnati, E.; Sartorio, G. Leader Progression Model of the lightning: Updating of the Model on the Basis of Recent Test Results. In Proceedings of the International Conference on Lightning Protection, Florence, Italy, 23-27 September 1996; pp. 399-407.

8. Dellera, L.; Garbagnati, E. Lightning stroke simulation by means of the leader progression model. I. Description of the model and evaluation of exposure of free-standing structures. IEEE Trans. Power Deliv. 1990, 5, 2009-2022. [CrossRef]

9. Rizk, F.A.M. Modeling of transmission line exposure to direct lightning strokes. IEEE Trans. Power Deliv. 1990, 5, 1983-1997. [CrossRef]

10. Bank Tavakoli, M.; Vahidi, B. Statistical analysis of the lightning performance of high voltage OHLs using dynamic simulation of lightning leaders movements. Int. J. Electr. Power Energy Syst. 2010, 32, 1024-1030. [CrossRef]

11. Kumar, U.; Bokka, P.K.; Padhi, J. A macroscopic inception criterion for the upward leaders of natural lightning. IEEE Trans. Power Deliv. 2005, 20, 904-911. [CrossRef]

12. Becerra, M.; Cooray, V. A self-consistent upward leader propagation model. J. Phys. D Appl. Phys. 2006, 39, 3708-3715. [CrossRef]

13. Cooray, V.; Arevalo, L. Modeling the stepping process of negative lightning stepped leaders. Atmosphere 2017, 8, 245. [CrossRef]

14. Campos, L.; Saba, M.; Warner, T.; Pinto, O.; Krider, E.; Orville, R. High-speed video observations of natural cloud-to-ground lightning leaders-A statistical analysis. Atmos. Res. 2014, 135-136, 285-305. [CrossRef]

15. Shah, W.; He, H.; He, J.; Yang, Y. Continuous and discontinuous streamer leader propagation phenomena under slow front impulse voltages in a 10-meter rod-plane air gap. Energies 2018, 11, 2636. [CrossRef]

16. Long, M.; Becerra, M.; Thottappillil, R. Modeling the Attachment of Lightning Dart and Dart-Stepped Leaders to Grounded Objects. IEEE Trans. Electromagn. Compat. 2017, 59, 128-136. [CrossRef]

17. Nag, A.; Rakov, V. A unified engineering model of the first stroke in downward negative lightning. J. Geophys. Res. 2016, 121, 2188-2204. [CrossRef]

18. Wang, D.; Takagi, N.; Uman, M.; Jordan, D. Luminosity progression in dart-stepped leader step formation. J. Geophys. Res. 2016, 121, 14612-14620. [CrossRef]

19. Wang, Y.; Qie, X.; Wang, D.; Liu, M.; Su, D.; Wang, Z.; Liu, D.; Wu, Z.; Sun, Z.; Tian, Y. Beijing Lightning Network (BLNET) and the observation on preliminary breakdown processes. Atmos. Res. 2016, 171, 121-132. [CrossRef]

20. Zhu, Y.; Rakov, V.; Tran, M. A study of preliminary breakdown and return stroke processes in high-intensity negative lightning discharges. Atmosphere 2016, 7, 130. [CrossRef]

21. Rakov, V.A.; Uman, M.A. Lightning: Physics and Effects; Cambridge University Press: Cambridge, UK, 2003. 
22. Golde, R.H. Lightning Protection; Chemical Publishing Company: New York, NY, USA, 1975.

23. Golde, R.H. Lightning, Volume 1: Physics of Lightning; Academic Press: New York, NY, USA, 1978.

24. Berger, K. Novel observations on lightning discharges: Results of research on Mount San Salvatore. J. Frankl. Inst. 1967, 283, 478-525. [CrossRef]

25. Hutzler, B. Notes Bibliographiques Concernat la Simulation en Laboratoire des Points d'Impact de la Foudre; Technical Report, Note Technique EDF, Ref. HM80-1173; Electricite de France: Paris, France, 1988. (In French)

(C) 2019 by the authors. Licensee MDPI, Basel, Switzerland. This article is an open access article distributed under the terms and conditions of the Creative Commons Attribution (CC BY) license (http:/ / creativecommons.org/licenses/by/4.0/). 